\title{
EXPLOSIVE SEISMIC SOURCES FOR THE MOON $\dagger$
}

\author{
ROBERT L. KOVACH* AND THOMAS J. AHRENS
}

The coupling of seismic energy under vacuum conditions, such as the moon, using an untamped surface charge is different from coupling in air. In vacuum, the explosive gas blast and the detonation products continuously expand outward and interact with the solid suriace. A series of model experiments was performed to investigate the effect of vacuum on coupling seismic energy. H.XS charges of $0.2 \mathrm{gm}$ each were detonated in contact with a plate and block of acrylic plastic in vacuum and in air. The amplitudes of the first and second arrivals (longitudinal and shear plate wave) are about 50 percent greater in vacuum than in air

\section{INTRODUCTION}

One of the geophysical experiments that is planned for performance on the moon as part of the Apollo manned landings is a seismic refraction experiment. The objectives of such an experiment are to investigate the near-surface elastic properties of the moon (Kovach and Press, 1962; Kovach, 1967).

Explosives have long been used on earth as the source of seismic signals, but their use on the lunar surface is not so straightforward. What explosive will survive exposure to the lunar environment and still reliably detonate? Since it is also impractical, at least for early missions, to bury the explosive charges, surface detonations will be used. Would small, untamped surface charges couple usable seismic signals in to the lunar media? Would the expanding mass of detonation gas induce signals of such frequency, amplitude, and traveltime as to obscure the desired ground arrivals? Does the absence of an atmosphere influence the generated seismic energy? Clearly the because the plate velocity $(\sim 2.4 \mathrm{~km} / \mathrm{sec})$ more closely matches the gas-blast velocity $(\sim 3.5$ to $7.5 \mathrm{~km} / \mathrm{sec})$ than the sound-wave velocity $(\sim 0.35$ $\mathrm{km} / \mathrm{sec}$ ). When the charges are detonated in contact with the block to generate direct body waves, little difference is noted in the first arrival amplitudes in air and vacuum; suspending the charge one charge-diameter above the surface produces about 25 percent lower first amplitudes in a vacuum. Large scale experiments were also performed in air to examine the effect of the detonation configuration on seismic coupling.

answers to these and closely related questions are of paramount importance.

When a seismic explosive charge is detonated in the atmosphere in contact with or in close proximity to the earth's surface, a seismic signal is generated in the surface material by the direct interaction of the high-pressure explosive gases or by interaction of the down-traveling air shock with the surface. Simultaneously, an air shock which initially travels at approximately $4 \mathrm{~km} / \mathrm{sec}$ propagates outward and upward into the air. The amplitude of the air shock attenuates rapidly to the low values of pressure commonly associated with sound waves. At normal temperatures the sound wave travels at $0.345 \mathrm{~km} / \mathrm{sec}$. Both the outward-expanding explosive gases and possible solid fragments are quickly brought to rest by the surrounding blanket of air. Meanwhile, the seismic signals induced in the surface material by the explosive charge propagate outward at a speed (compressional-wave velocity) varying from a fraction of a kilometer per second to

$\dagger$ Division of Geological Sciences Contribution No. 1621, California Institute of Technology, Pasadena, California. Manuscript received by the Editor August 14, 1969. Revised manuscript received November 6, 1969.

* Department of Geophysics, Stanford University, Stanford, California 94305.

‡ Seismological Laboratory, California Institute of Technology, Pasadena, California 91109.

Copyright (C) 1970 by the Society of Exploration Geophysicists. 
approximately $9 \mathrm{~km} / \mathrm{sec}$ (for rocks rich in ferro magnesian silicates).

In a refraction experiment the seismometers detect signals at successively greater distances. These seismic signals will have propagated via successively deeper subsurface paths. The apparent velocity with which the first signals are detected by the seismometer approaches, at sufficiently great distances, the compressionalwave velocity of the rock below. Since the compressional-wave velocity of rocks is generally higher than that of the sound wave in air, the seismic signals from the ground are recorded well before the sound wave arrives and disturbs the seismometers.

In the lunar vacuum the situation in regard to the explosive gases is quite different from that in the earth's atmosphere. Upon detonation of the charge, some of the expanding explosive gases will interact with the surface and induce a seismic signal into the surface material. The explosive gases which do not interact with the suriace will expand outward freely into the lunar vacuum. Initially they will travel at 2 to $3 \mathrm{~km} / \mathrm{sec}$, a velocity corresponding to the detonation state in the explosive. In the outer layer of explosive gases, as expansion occurs, the kinetic energy, and hence also the gas velocity, increase rapidly at the expense of the gas internal energy; the latter decreases rapidly (Ahrens et al, 1970). As a result, the explosive product gases can sweep along the surface at very high velocity (experimental results indicate a speed of $10 \mathrm{~km} / \mathrm{sec}$ ). This sweeping of the explosive gases across the lunar surface can give rise to at least three phenomena:

1. As the explosive-gas blast sweeps along the lunar surface at a supersonic velocity with respect to the lunar material, it acts as a radially expanding source of seismic energy. A seismometer some distance away may detect a seismic signal, induced by these expanding gases arriving at the seismometers well before the desired seismic signal originating at the point of detonation. Thus a velocity may be measured that is significantly higher than the actual elastic velocity of the lunar material.

2. Fven after expanding outward for some hundreds of meters from the charge, the explosive gases may, upon direct interaction, impart sufficient relocity to the highly sensitive scismometers to produce a detectable signal. Since the velocity of the gas blast is comparable to a velocity slightly higher than the elastic velocity in common rocks, such a signal may interfere with or even mask the seismic signals from below.

3. Because the explosive-gas blast rapidly covers a large area of the lunar surface, it may induce a seismic signal for which the energy distribution among different modes of wave propagation differs significantly from the energy distribution induced in air by a point-source explosion.

In this paper we briefly discuss the selection of an explosive for lunar geophysical use, and the results of a set of small-scale laboratory measurements which were performed to examine the relative efficiency of seismic coupling in vacuum and air together with the close-in effects of the exploding gas blast on conventional seismometers. In addition, a series of larger-scale experiments was performed in air to examine the ground amplitudes generated by small surface charges detonated in different configurations.

\section{CHOICE OF EXPLOSIVE}

The environmental conditions of the Moon to which an explosive must be exposed and still detonate reliably are severe. These conditions include exposure to a hard vacuum of at least $10^{-11}$ Torr, a temperature range of $100^{\circ} \mathrm{K}$ to $400^{\circ} \mathrm{K}$, and a 28-day temperature cycle. Any material selected must, therefore, have a low vapor pressure and high resistance to temperature extremes and thermal gradients.

Explosives capable of storage and operation at extreme temperatures are often required in missile and aircrait applications. However, in the years following World War II the choice of hightemperature explosives was primarily limited to RDX and HMX. These explosives will survive exposure to the Iunar temperatures but are extremely sensitive to detonation under shock and could not be considered for geophysical use by astronauts on the moon. RDX would also sublime rapidly in the lunar environment.

Research into other high temperature explosives such as I)ipam (diamino-hexanit robiphenyl) and HNS (hexanitrostilbene) was carried out beginning in 1955 by the Naval Ordnance Laboratory in Maryland. Examination of the properties of these explosives led to the choice of HAS-II with 10-percent 'Teflon as the binding agent as 
Table 1. Properties of high temperature explosives

\begin{tabular}{|c|c|c|c|c|c|c|c|}
\hline Property & TNT & HMX & $\mathrm{RDX}$ & TACOT & DАTB & Dipam $^{c}$ & HNS-IJe \\
\hline $\begin{array}{l}\text { Melting } \\
\text { point }{ }^{\circ} \mathrm{C}\end{array}$ & 81 & 285 & 204 & 410 & 295 & 304 & 318 \\
\hline $\begin{array}{l}\text { Detonation } \\
\text { velocity } \\
\mathrm{mm} / \mu \sec (a \rho \\
\mathrm{gm} / \mathrm{cm}^{3}\end{array}$ & $6.93 @ 1.63$ & $9.11 @ 1.89$ & $8.64(a 1.77$ & 6.60 & $7.52(a) 1.79$ & $7.4(a 1.79$ & $7.0 \Leftrightarrow 1.70$ \\
\hline $\begin{array}{l}\text { Vacuum } \\
\text { stability }(a) \\
260^{\circ} \mathrm{C}, \mathrm{cm}^{3} / \mathrm{gm} / \mathrm{h}\end{array}$ & & & $13.2\left(\alpha 180^{c}\right.$ & & & 1.26 & 0.23 \\
\hline $\begin{array}{l}\text { Vapor pressure } \\
\mathrm{mm} \mathrm{Hg} a^{\circ} \mathrm{C}\end{array}$ & $0.106(a 100$ & & $0.026(a) 162^{\mathrm{e}}$ & & & & $3.9 \times 10^{-8}(a) 162$ \\
\hline $\begin{array}{l}\text { Impact } \\
\text { sensitivity } \\
\text { explosion } \\
\text { height, cm, } \\
2.5 \mathrm{~kg} \text { weight }\end{array}$ & 100 & 30 & 28 & $142-5 \mathrm{~kg}$ & $>17 i$ & 95 & 63 \\
\hline $\begin{array}{l}\text { Gap test sensi- } \\
\text { tivity, mils }\end{array}$ & 13 & & 204 & & 14 & & \\
\hline \multicolumn{8}{|c|}{$\begin{array}{l}\text { Lawrence Radiation Lab. (1965) } \\
\text { b Thatcher, D. N. (1965) } \\
\text { ' Kilmer, E. E. (1968) } \\
\text { d Drop weight tests. Values }<25 \mathrm{~cm} \text { quite sensitive to impact; values }>70 \mathrm{~cm} \text { relatively insensitive. } \\
\text { e Measure of shock sensitivity. The larger the value the more sensitive to shock. }\end{array}$} \\
\hline
\end{tabular}

the candidate explosive for lunar geophysical use (see Table 1). HNS-II exposed in outer space is calculated to recede only $0.01 \mathrm{~cm} / \mathrm{yr}$ at $150^{\circ} \mathrm{C}$ in contrast to an RID surface which would recede $2.5 \mathrm{~cm} / \mathrm{hr}$ under the same conditions (Kilmer, 1968). HNS-II also has excellent thermal properties.

\section{THE EFFECT OF VACUUM ON SEISMIC COUPLING}

Small-scale laboratory measurements of explosive coupling in vacuum and air were performed to examine the relative effectiveness of small, untamped surface charges. In one set of experiments, pellets of pure HNS (200 mg) were detonated in contact with a $2.5 \mathrm{~cm}$-thick plate of Plexiglas. The pellets were detonated at one end using a confined mild detonating fuse; the fuse was detonated using a bridgewire detonator. The plate was mounted in a horizontal position and the charges and detectors were placed on the top surface of the plate so that a three-dimensional model was obtained (Figures 1 and 2).

Plexiglas was chosen as the model material because of the ease with which a hard vacuum may be achieved in a chamber containing a relatively large quantity of this material and because of the similarity of its low shock impedence to that of porous rock. The seismic velocities of Plexiglas are also low enough that easily measurable traveltimes can be attained in small-scale experiments. The parameters of Plexiglas are (Oliver et al, 1954):

Body dilatational velocity $2.7 \mathrm{~mm} / \mu \mathrm{sec}$, Plate dilatational velocity $2.36 \mathrm{~mm} / \mu \mathrm{sec}$,

Body shear velocity $1.37 \mathrm{~mm} / \mu \mathrm{sec}$, Density $1.22 \mathrm{gm} / \mathrm{cm}^{3}$.

The seismic signals generated in the Plexiglas were recorded at a distance of about $40 \mathrm{~cm}$ from the point of explosive detonation, using a piezoelectric (PZT) crystal and a conventional HallSears $7.5 \mathrm{~Hz}$ vertical-component geophone. The outputs of the transducers were observed on calibrated oscilloscopes.

The results obtained are summarized in Table 2. The PZT crystal and the geophone detected different first-wave arrivals; these had velocities which corresponded closely with the body-and plate-dilatational velocities of the plate. ${ }^{1}$ It

1 The governing equations for a homogeneous plate bonded by two varallel planes are well known. Symmetric and antisymmetric vibrations are possible. For 


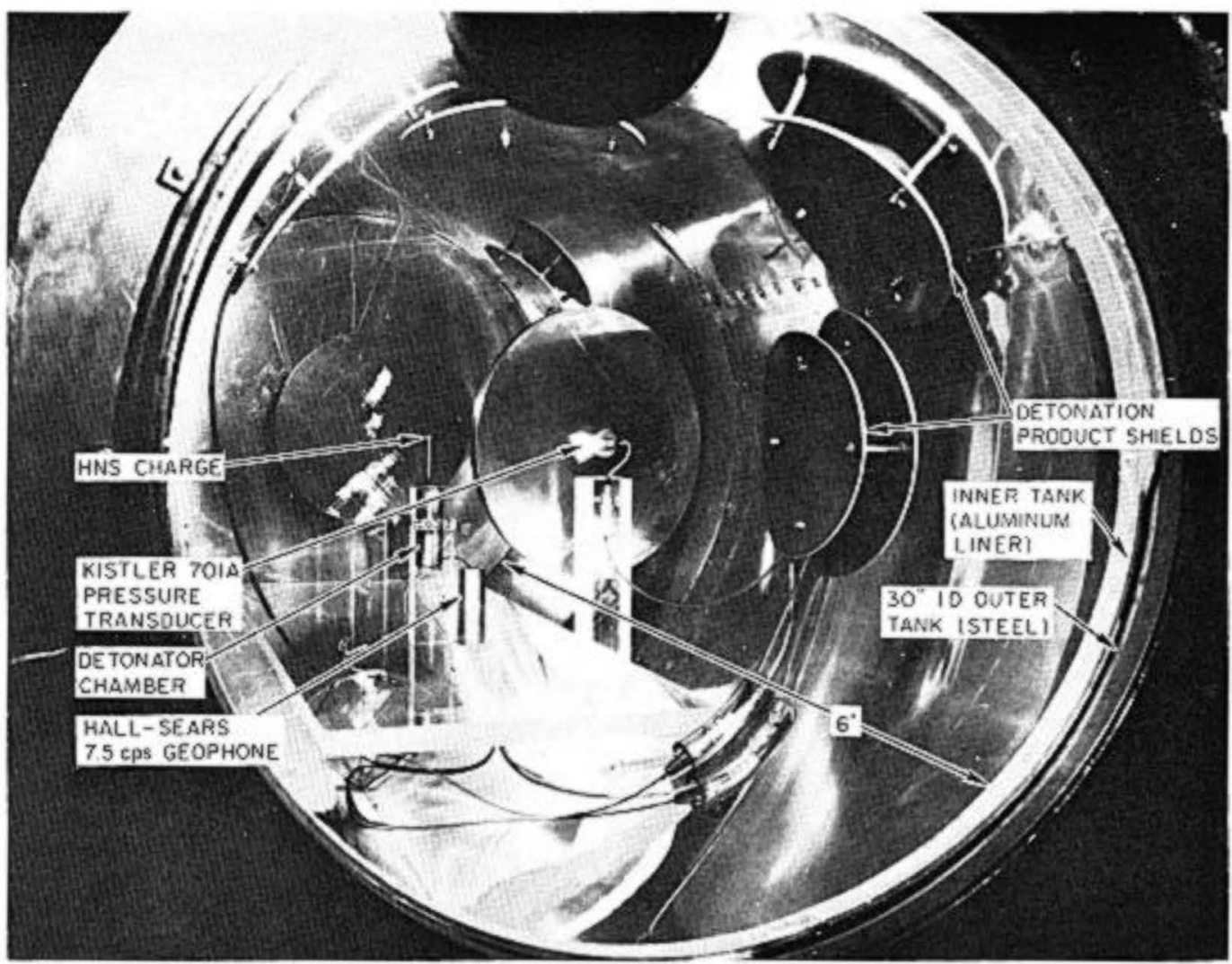

FIG. 1. Experimental arrangement for measuring explosive-gas blast in vacuum tank. Geophone is bolted directly to Plexiglas plate.

should be pointed out that the arrival times are somewhat shorter than those predicted by elastic wave theory (higher apparent velocities). Near the explosion point a shock wave (higher velocity) rather than an elastic wave description of the seismic energy propagation is undoubtedly more appropriate; in these experiments we did not attempt to delineate the highly stressed zone from the elastic region.

The peak-to-peak amplitude of the first arrival at the geophone was used as the quantitative measure of the coupling efficiency for the small explosive charges. The amplitudes of the first and second arrivals are about $\mathbf{5 0}$ percent greater in vacuum than in air. Figure 3 is a plot of the arrival time of the peak pressure induced upon

waves long compared to the thickness of the plate the period equation for symmetric vibrations degenerates to the form for plate waves; for waves short compared to the thickness of the plate the equation approaches the period equation for Rayleigh waves on a solid. reflection of the explosive-gas blast as a function of distance for a $0.2 \mathrm{gm}$ HNS charge (Ahrens et al, 1970). The figure shows that out to the experimental distances of interest $(\sim 40 \mathrm{~cm})$ the peak pressure of the gas blast travels at a velocity of 3.5 to $7.5 \mathrm{~mm} / \mu \mathrm{sec}$. This is much faster than the speed of sound in air $(0.345 \mathrm{~km} / \mathrm{sec})$ and is closer to the plate velocity of Plexiglas. We therefore conclude that for comparable surface-detonated charges the compressional plate wave is more easily excited under vacuum conditions than in air. The amplitude of the second arrivals, which may represent the plate shear wave, also appear to be enhanced in vacuum. These results suggest that, with all other factors remaining the same, on the moon it may be somewhat easier to excite seismic surface waves with surface detonation charges than on the earth.

Since the first set of experiments was concerned with waves traveling along a plate, a 


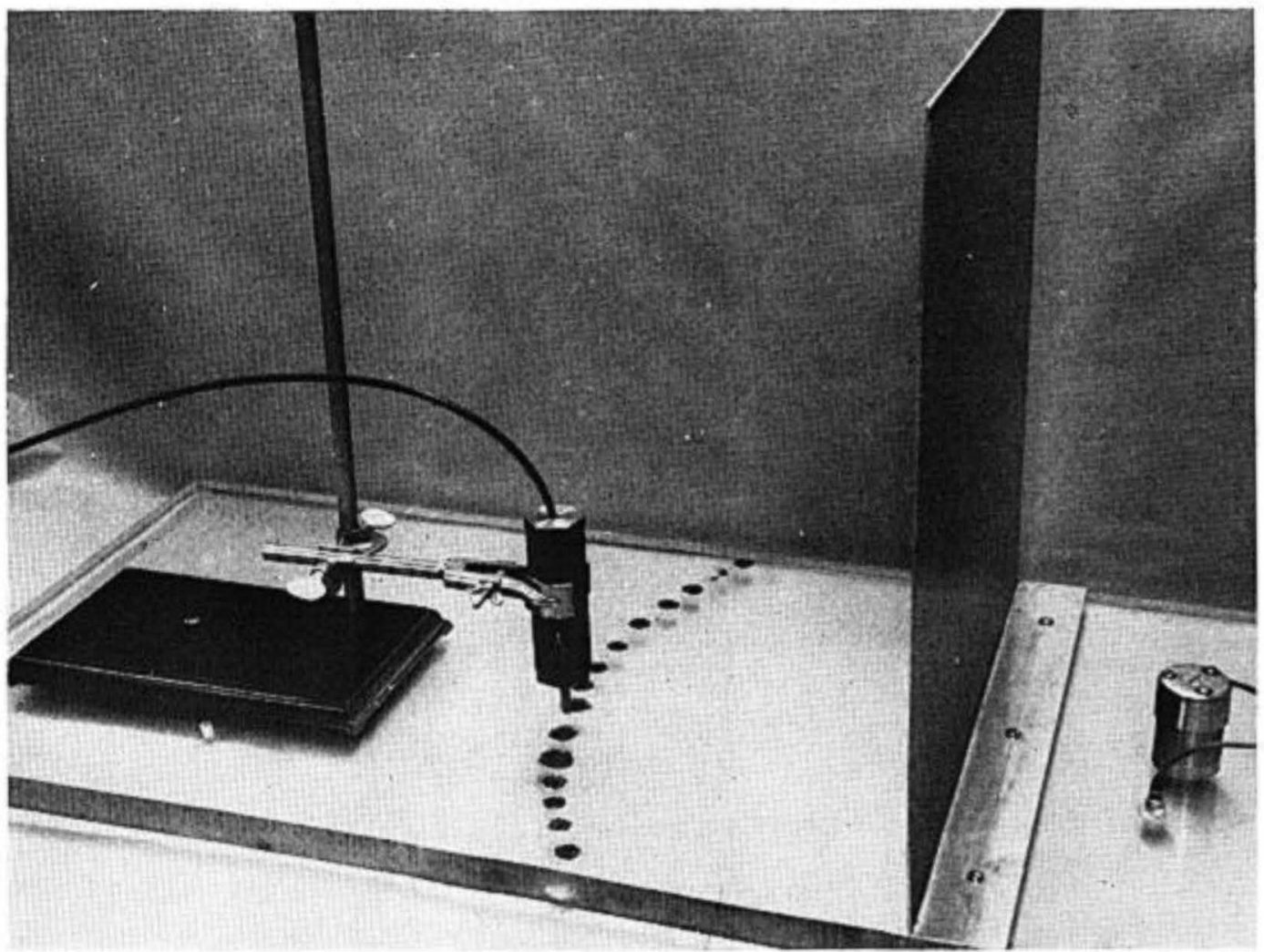

lig. 2. Plate configuration for small-scale seismic coupling experiments. Metal shield prevents explosive-gas blast from interacting with geophone.

Table 2. Results of small-scale coupling experiments on a Plexiglas plate

\begin{tabular}{|c|c|c|c|c|c|c|c|}
\hline Shot no. & $\begin{array}{l}\text { Distance } \\
\text { from shot } \\
\text { to PZT } \\
\text { crystal } \\
(\mathrm{mm})\end{array}$ & $\begin{array}{c}\text { Velocity } \\
\text { to PZT } \\
(\mathrm{mm} / \mu \mathrm{sec})\end{array}$ & $\begin{array}{c}\text { First } \\
\text { arrival } \\
\text { velocity } \\
(\mathrm{mm} / \mu \mathrm{sec})\end{array}$ & $\begin{array}{c}\text { Second } \\
\text { arrival } \\
\text { velocity } \\
(\mathrm{mm} / \mu \mathrm{sec})\end{array}$ & $\begin{array}{c}\text { First } \\
\text { arrival } \\
\text { amplitude } \\
(\mathrm{mv})\end{array}$ & $\begin{array}{c}\text { Second } \\
\text { arrival } \\
\text { amplitude } \\
(\mathrm{mv})\end{array}$ & $\begin{array}{c}\text { Pressure } \\
\text { (torr) }\end{array}$ \\
\hline 4 & 366 & 2.88 & - & - & 105 & - & Ambient \\
\hline 5 & - & 2.00 & 2.47 & 1.27 & 110 & 1600 & Ambient \\
\hline 8 & 386 & 2.88 & 2.49 & 1.28 & 105 & 1660 & Ambient \\
\hline 9 & - & - & 2.44 & 1.28 & 90 & 1550 & Ambient \\
\hline 10 & 389 & 2.88 & - & - & - & - & Ambient \\
\hline 11 & 395 & 2.92 & 2.47 & 1.31 & 106 & 2050 & $1.2 \times 10^{-4}$ \\
\hline 13 & 400 & 2.88 & 2.32 & - & 156 & 3000 & $1.2 \times 10^{-4}$ \\
\hline 24 & 405 & 2.77 & 2.44 & 1.31 & 160 & - & $5.6 \times 10^{-5}$ \\
\hline 25 & 410 & 2.75 & 2.43 & 1.30 & 180 & - & $4.5 \times 10^{-5}$ \\
\hline 32 & 418 & 2.84 & - & - & 120 & $\ldots$ & Ambient \\
\hline 30 & 359 & 2.82 & 2.41 & - & 115 & $\ldots$ & Ambient \\
\hline 31 & 3.53 & 2.83 & 2.39 & - & 100 & - & Ambient \\
\hline
\end{tabular}

s $0.2 \mathrm{gm} \mathrm{HNS}$ charges, $6.4 \mathrm{~mm}$ diameter, $1.6 \mathrm{gm} / \mathrm{cm}^{3}$.

b Distance to geophone fixed at $381 \mathrm{~mm}$. 


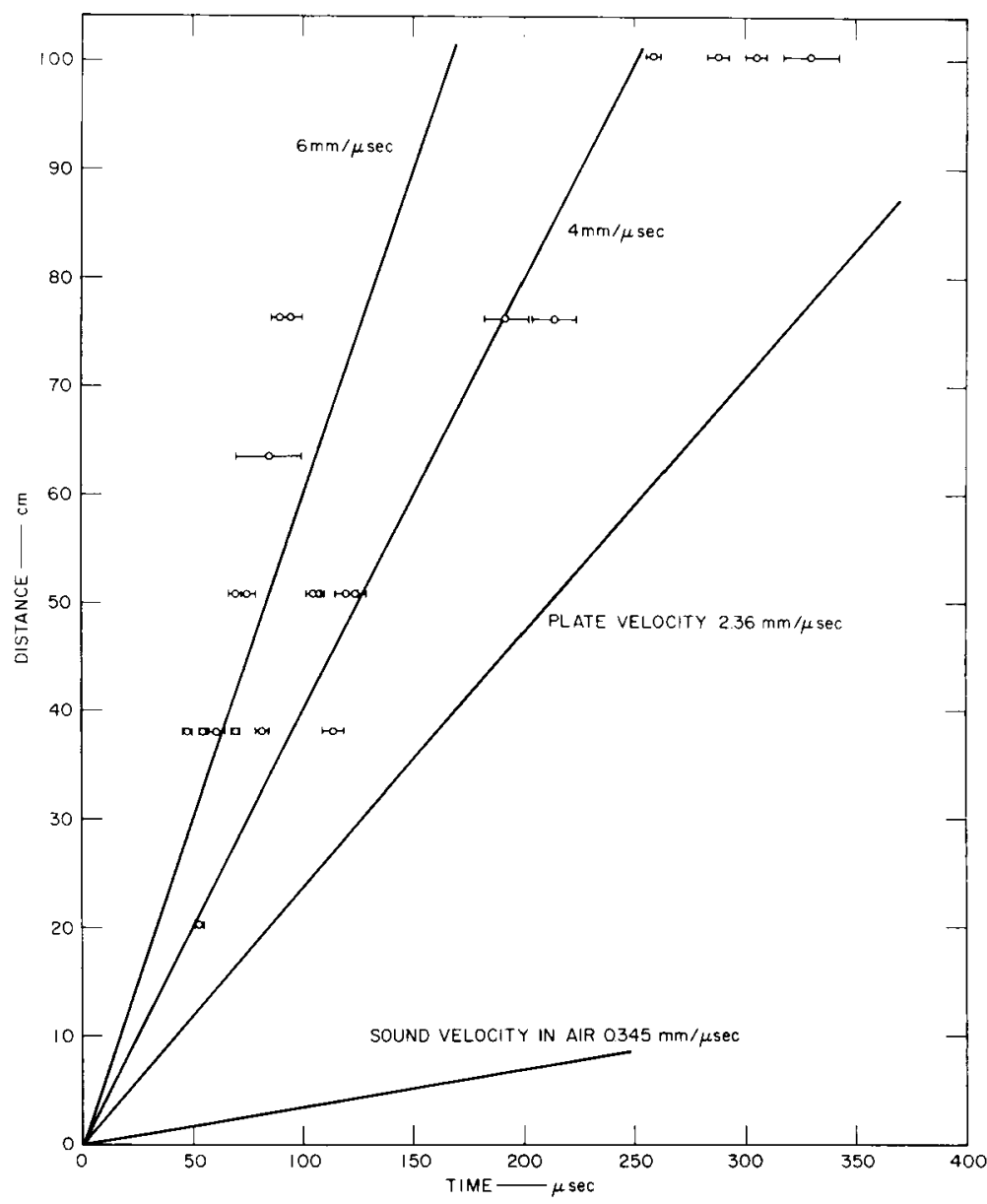

l'IG. 3. Distance to transducer versus time of arrival of peak gas-blast pressure.

second series of small-scalc expcriments was designed to compare the efficiency of coupling seismic energy into direct body waves through a block from small charges detonated on and slightly above its surface. A Plexiglas block 10 $\mathrm{cm}$ thick (Figure 4) was employed as the model in these experiments. The charges were $0.201 \mathrm{gm}$ of $\mathrm{H} \times \mathrm{S}$ detonated in contact with or slightly offset from the thick Plexiglas block. A $7.5 \mathrm{~Hz}$ HallSears geophone was positioned on the opposite side of the block to record the first direct seismicwave arrival.

The experimental results (Table 3) demonstrate that the amplitude of the first arrival in air differs little from that in vacuum when the explosive charge is positioned directly against the surface (shots $40,42,45,48,54$ ). However, the signal amplitudes appear to be significantly and reproducibly lower (by approximately 25 percent) for the vacuum shots compared with those fired in air when the explosive charge is suspended one charge-diameter above the surface of the block (compare Table 3 data for shots 44,46 , and 49 with 47,50 , and 55).

Comparison of the results obtained in air when the charge is detonated in contact with the block with those when it is suspended one chargediameter above the block reveals that in the latter case there is approximately a 20 to 30 percent reduction in the observed amplitude. A similar experiment performed in a vacuum (shots $42,47,50,54,55,56)$ shows that the observed 


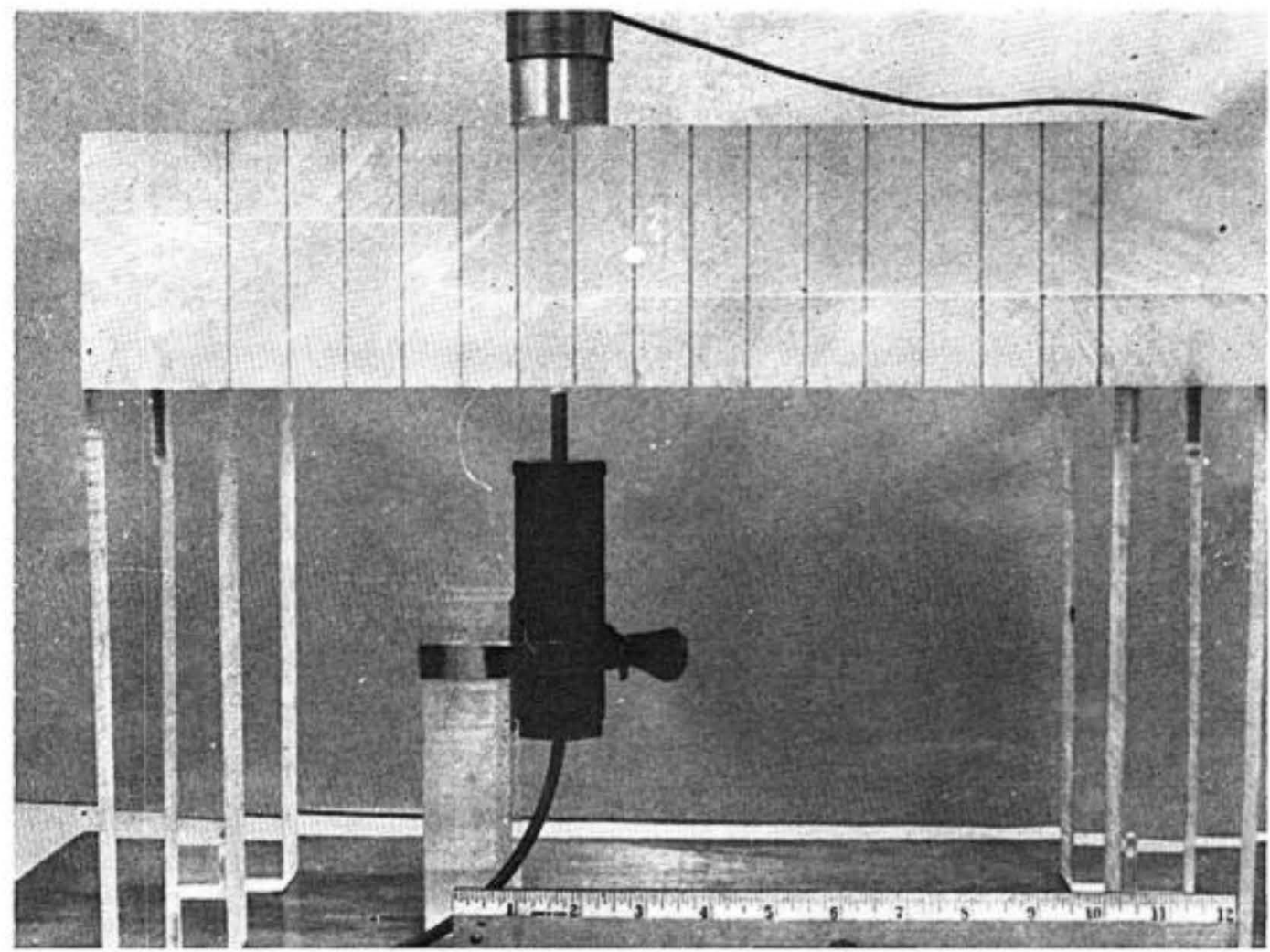

FIG. 4. Configuration of small-scale body wave seismic coupling experiments.

amplitudes for the suspended charge are about 40 percent less than those obtained when the charge is detonated in contact with the block.

\section{DIRECT EFFECT OF EXPLOSIVE-GAS BLAST ON GEOPHONES}

The direct response of a geophone to gas blast in a vacuum was investigated in the course of performing explosive-gas blast measurements. A $7.5 \mathrm{~Hz}$ geophone was mounted on a 2.5 -cm-thick Plexiglas plate and placed at distances ranging from 38 to $102 \mathrm{~cm}$ from the center of 0.2 and 2.6 gm HNS charges in the vacuum chamber (Figure 1). The voltage signal (proportional to velocity) generated by the geophone was recorded directly with oscilloscopes.

Since the height of the center of the charge above the Plexiglas plate was held constant at 27 $\mathrm{cm}$ and the slant distance to the geophone from the center of the charge was varied from 38 to $102 \mathrm{~cm}$, the flow direction of the gas blast varied from $35^{\circ}$ to $15^{\circ}$ from the horizontal. The maxi- mum geophone voltages were observed between 400 and $1000 \mu \mathrm{sec}$ after the charge was detonated, depending on the distance from the charge to the seismometer. The exact time at which the gas blast signal arrives at the geophone is difficult to detect (Figure 5) because of the poor response of the seismometers above $\sim 1 \mathrm{kHz}$. [This characteristic of the geophone is expected because mechanical wave propagation effects in the geophone case (i.e., several centimeters long) effectively determine the upper limit of the frequency response.] For the relatively short distances over which measurements were made, the apparent velocity of the signal to which the seismometer initially responds varies from 2.5 to 7 $\mathrm{km} / \mathrm{sec}$. Most of the measurements indicated an apparent velocity in the range of 4 to $7 \mathrm{~km} / \mathrm{sec}$. Since the fastest mode of mechanical (longitudinal body) wave propagation in Plexiglas has a speed of $2.7 \mathrm{~km} / \mathrm{sec}$, it can be concluded that the signal recorded on the geophone is a superseismic phenomenon and is probably the result of 
Table 3. Vacuum coupling through a Plexiglas block

\begin{tabular}{|c|c|c|c|c|c|}
\hline Shot no. & $\begin{array}{l}\text { HNS charge } \\
\text { weight } \\
\text { (gm) }\end{array}$ & $\begin{array}{l}\text { Thickness of } \\
\text { Plexiglas } \\
\text { (mm) }\end{array}$ & $\begin{array}{l}\text { Offset } \\
(\mathrm{mm})\end{array}$ & $\begin{array}{l}\text { Pressure } \\
\text { (torr) }\end{array}$ & $\underset{\text { (volts) }}{\text { Amplitude }}$ \\
\hline 40 & 0.2020 & 101.0 & 0 & Ambient & 5.8 \\
\hline 41 & 0.2030 & 101.0 & 6.4 & Ambient & - \\
\hline 42 & 0.2020 & 101.0 & 0 & $4.1 \times 10^{-5}$ & 6.8 \\
\hline 43 & 0.2000 & 101.0 & 6.4 & Ambient & - \\
\hline 44 & 0.2040 & 101.0 & 6.4 & Ambient & 4.3 \\
\hline 45 & 0.2025 & 100.5 & 0 & Ambient & 6.4 \\
\hline 46 & 0.2010 & 100.5 & 6.4 & Ambient & 4.6 \\
\hline 47 & 0.1990 & 100.5 & 6.4 & $4.1 \times 10^{-5}$ & 3.7 \\
\hline 48 & 0.2020 & 100.5 & 0 & Ambient & 6.5 \\
\hline 49 & 0.2015 & 100.3 & 6.4 & Ambient & 4.2 \\
\hline 50 & 0.2020 & 100.25 & 6.4 & $3.8 \times 10^{-5}$ & 3.4 \\
\hline 54 & 0.2035 & 100.25 & 0 & $3.9 \times 10^{-5}$ & 7.0 \\
\hline 55 & 0.2025 & 100.25 & 6.4 & $3.6 \times 10^{-5}$ & 3.6 \\
\hline 56 & 0.1990 & 100.25 & 0 & $2.5 \times 10^{-5}$ & 6.8 \\
\hline
\end{tabular}

s $6.4 \mathrm{~mm}$ diam. pellet; density $1.68 \mathrm{gm} / \mathrm{cm}^{3}$

the arrival of an explosive gas blast at the geophone case.

In sume experiments a small precursor signal preceded the large main signal, indicating that the geophone was reacting to a velocity induced from the Plexiglas plate below. The apparent speed of this precursor signal was comparable to that of the gas blast itself. This signal is probably the result of the gas-blast front inducing a superseismic signal in the Plexiglas as it sweeps along the surface.

It is important that these experimental data be scaled in some manner to predict the effects at larger distances. One common scaling variable is the mass of the charge. For a sphere of explosive of constant density a characteristic length associated with the expansion of the detonation products is the initial radius of the explosive charge. Mass is proportional to the cube of the radius; thus, when comparing the effects of two different mass charges $W_{1}$ and $W_{2}$, a characteristic distance should scale by a factor $k$ such that $k^{3}$ $=W_{2} / W_{1}$. This behavior is often called $W^{1 / 3}$ scaling.

The voltage induced in the geophones (predominant frequency $\sim$ one $\mathrm{kHz}$ ) by the direct gas blast (Figure 6) lie along a curve for which the voltage may be scaled by $W^{1 / 3}$ scaling. By using a measured high-frequency calibration factor of $0.6 \mathrm{v} / \mathrm{cm} / \mathrm{sec}$ for the geophone used in the experiment, these voltage data may be converted to geophone velocity (centimeters per second). Since the experimental data appear to lie along a straight line in the log velocity-log radius plane, the following law for geophone velocity from a 1-lb charge of HNS is suggested

$$
v=a r^{b},
$$

where $a=301$ and $b=-445$. Hence $v$ is in centimeters per second and $r$ is in meters. Extrapolation of these data over $1-1 / 2$ orders of magnitude in scale distance indicates that a voltage of approximately $10 \mu \mathrm{v}$ will be induced by direct action of an explosive-gas blast in a vacuum from a 454 $\mathrm{gm}(1 \mathrm{lb})$ charge at a distance of approximately $100 \mathrm{~m}$.

\section{SEISMIC COUPLING AND DETONATION CONFIGURATION EXPERIMENTS}

Surface-detonated charges are commonly used for seismic refraction shooting in the Sahara. Buffet and Layat (1960) in a comprehensive study 
examined the effects of charge shape and location of the detonating mechanism, the influence of the height of suspension of explosive charges above the ground, and the influence of charge weight on the recorded seismic-wave amplitudes in the distance range from 1250 to $3000 \mathrm{~m}$. Their pertinent results are summarized as follows:

(a) For cylindrical charges weighing $6.25 \mathrm{~kg}$ detonated $3 \mathrm{~m}$ above the ground, the effect of the location of the priming mechanism within the charges gave variations in the recorded ground amplitudes of less than 10 percent.

(b) A 40-percent increase in recorded ground amplitudes was measured when the height above the ground of the explosive charges (ranging in weight from $3.125 \mathrm{~kg}$ to $25 \mathrm{~kg}$ ) was $3 \mathrm{~m}$ as compared to detonation on the surface.

Because the sizes of the explosive charges used in their study are larger than can be used in early lunar experiments, a set of field experiments was performed using much smaller explosive weights. These experiments were designed to assess the effects of the detonation configuration on seismic coupling.

Cylindrical charges having a length-to-diameter ratio of unity were cast from pure TNT. The charges ranged in weight from about $57 \mathrm{gm}$ to $454 \mathrm{gm}(1 / 8$ to $1 \mathrm{lb})$ and ranged in diameter from 3.53 to $7.04 \mathrm{~cm}$. The explosive charges were placed at distances ranging from 66 to $580 \mathrm{~m}$ from the recording geophone spread and detonated with the booster placed at different positions. Three configurations were used. In the first, the booster was placed at the top of the cylindrical charge with the bottom of the cylinder resting on the ground; detonation occurred from the top to the bottom. In the second, the booster was placed on the bottom of the cylinder so that detonation occurred from the bottom up. The third configuration involved suspending the change one diameter above the ground and detonating it from the top down. The amplitude of the first seismic arrival was defined as the amplitude of the second half-cycle in the first wave motion to reach the geophones.

The experimental data revealed that for the experiments performed on a gravelly soil at atmospheric pressure there can be as much as 50 -per-

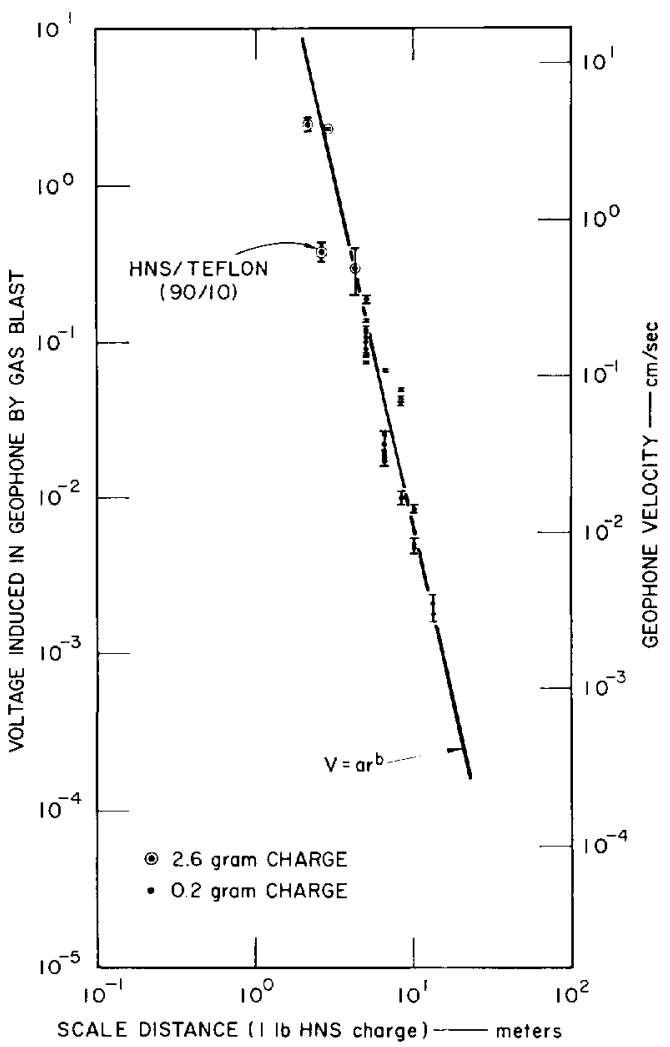

FIg. 5. Voltage signal induced by explosive-gas blast striking 7.5-Hz Hall-Sears geophone $64 \mathrm{~cm}$ from 0.2 gm HNS charge.

cent increase in recorded ground amplitudes for small surface-detonated cylindrical charges, depending on the orientation of the initiation with respect to the ground (i.e., top or bottom).

Because it was uncertain what the effects of the test medium (i.e. gravelly soil) were on the recorded seismic amplitudes a similar set of field experiments was performed on a homogeneous dry-lake playa. In addition, comparison data were needed between the seismic energy induced in the ground as the result of detonaling 1-lb charges of HNS/Teflon $(90 \% / 10 \%)$ and conventional TNT. As before, the explosive charges were placed at various distances from the geophone spread and detonated with the letonators placed in different positions on the charges.

The values of seismic amplitude obtained on a dry-lake playa were closely rcproducible from shot to shot. A comparison of shots detonated from the top down with those detonated from the 


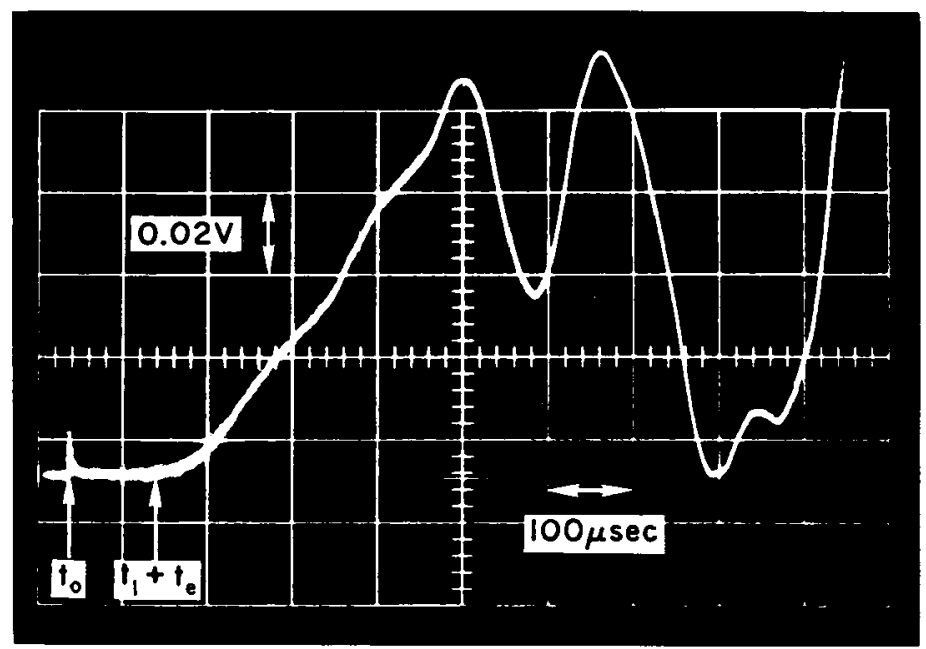

FIG. 6. Voltage induced in geophone by gas blast and corresponding geophone velocity versus scale distance for one-lb HNS charge.

bottom up revealed that there was little difference in the signals observed. 'Thus, in contrast to the results obtained on a gravelly soil, the ground motion measured on a dry-lake playa (finegrained material) was insensitive to the detonation configuration.

The amplitudes of the initial seismic arrivals recorded in the experiments on playa and gravelly soil with TNT and HNS are plotted in Figure 7 together with the signal amplitudes recorded from other near-surface conventional and nuclear explosions. A comparison of the amplitudes of the seismic signals, at similar distances, produced by HNS/Teflon $(90 \% / 10 \%)$ with those produced by comparable weight of 'TNT revealed that the HNS-generated amplitudes are only slightly less than those generated by $\mathrm{T} \times \mathrm{T}$. In the figure no account is taken of the test medium (except where noted) or of the recorded frequency of the first arrival. The charge weights and distances have been normalized to $1 \mathrm{lb}$ of TNT, using $W^{1 / 3}$ scaling. The data obtained on gravelly soil and playa at scale distances of less than $2000 \mathrm{ft}$, as well as the other data for conventional explosions obtained at shorter scale distances, lie along the band indicated in Figure 7. All these data apparently correspond to a direct wave path from the source to the receiver. The circled data, and probably the nuclear explosion results as well, correspond to high amplitude-refracted wave arrivals. The direct arrival data indicate amplitudes of 0.1 to $10 \mathrm{~m} \mu$ at the scale distances planned for early lunar seismic exploration; these are an order of magnitude lower than one would predict ( 10 to $100 \mathrm{~m} \mu$ ) from the nuclear data alone.

\section{SUMMARY AND CONCLUSIONS}

A series of model experiments were performed to investigate the effects of vacuum on coupling seismic energy from charges detonated on or slightly above the surface of a 10 -cm-thick Plexiglas block. When the charges were detonated in contact with the Plexiglas block, little difference was observed in the first arrival amplitudes in air and in vacuum. However, when the charges were suspended one charge-diameter above the surface, the initial seismic-signal amplitudes were about 25 percent lower in vacuum than in air.

In another series of experiments $0.2 \mathrm{gm} H \times \mathrm{S}$ charges were detonated in contact with a $2.5-\mathrm{cm}-$ thick Plexiglas plate. The peak-to-peak amplitudes of the longitudinal and shear plate wave arrivals at the geophone were used as a quantitative measure of the coupling efficiency in vacuum relative to that in air. The amplitudes of the plate-wave arrivals were about 50 percent greater in vacuum than in air. The more efficient coupling in vacuum than air observed for plate waves is believed to occur because of the closer agreement of the plate velocity to the gas-blast velocity than the sound-wave velocity. It thus may be easier 


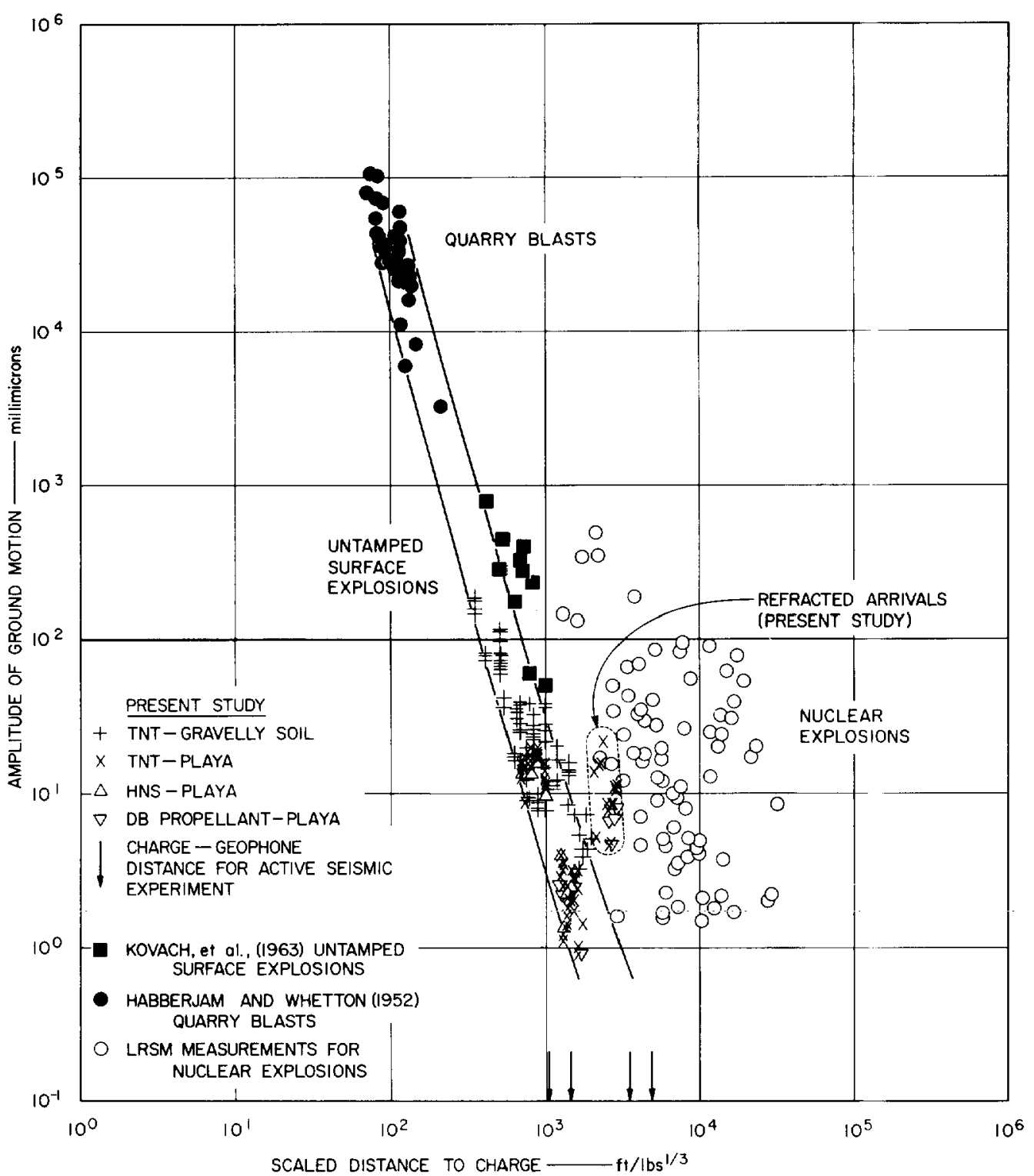

Fig. 7. Amplitude of first ground motion versus scale distance.

to excite seismic surface waves with surface charges on the moon than on the earth.

Signals are also generated in the geophones as a result of their direct interaction with the explosive-gas blast in a vacuum together with the generation of a small precursor signal traveling at a superseismic velocity. This superseismic signal results from the gas blast sweeping across the surface on which the geophones are mounted.
Large-scale experiments performed in air to examine the effects on seismic: coupling under different detonation configurations revealed that seismic amplitudes were dependent on the test media. For cylindrical explosive charges (diameter-to-height ratio $\sim 1$ ) detonated on the surface of a gravelly soil, the seismic signal was some 25 to 50 percent greater when the charge was initiated at the bottom than when the charge was 
initiated at the top. However, similar experiments performed on a dry-lake playa (very fine silt) showed no correlation of seismic amplitude with charge detonation point.

\section{ACKNOWLEDGMENTS}

This research was performed while the authors were staff members of Stanford Research Institute. We appreciate the able assistance of Charles F. Allen who participated in many of the experiments. Preparation of this report was supported by the National Aeronautics and Space Administration under grant NGL 05-020-2.32.

\section{REFERENCES}

Ahrens, T. J., Allen, C. F., and Kovach, R. L., 1970, - Explosive gas blast: The expansion of detonation products in vacuum: J. Appl. Phys., in preparation.

Buffet, A., and Layat, C., 1960, Touvel aspect de la sismique auréfraction Sahara: tirs non enterrés: Geophys. Prosp., v. 8, p. 47-67.
Habberjam, G. W., and Whetton, J. T., 1952, On the relationship between seismic amplitude and charge of explosives fired in routine blasting operations: Geophysics, v. 17, p. 116-128.

Kilmer, E. E., 1968, Heat resistant explosives for space applications: J. of Spacecra't and Rockets, v. 5, p. 1216-1219.

Kovach, R. I., 1967, Lunar seismic exploration, in The physics of the moon: Amer. Astronaut. Soc. Sci. and Tech. Ser., v. 3, p. 189-198.

and Press, F., 1962, Lunar seismology: Proc. Conf. on Lunar Exploration, Bull. 152, Virginia Polytechnic Institute, v. 56.

- Lehner, F, and Miller, R., 1963, Experimental ground amplitudes from small surface explosions: Geophysics, v. 28, p. 793-798.

Lawrence Radiation Laboratory, 1965, Properties of chemical explosives, Univ. Calif. UCRL-14592, Dec. 16.

Oliver, J., Press, F., and Ewing, M., 1954, Two-dimensional model seismology: Geophysics, v. 19, p. 202-219.

Thatcher, D. N., 1965, TACO'T- I new thermallystable explosive: E. I. duPont de Nemours and $C o$. Bull. 